\title{
Collaborating around the tabletop
}

\author{
Gerry Stahl
}

Published online: 10 November 2011

(C) International Society of the Learning Sciences, Inc.; Springer Science+Business Media, LLC 2011

In romantic visions of yesteryear, the idyllic nuclear family gathered around the kitchen table to share a bountiful dinner meal or the extended family came together around the dining-room table for a traditional holiday feast. These were occasions for lively and significant discussions, where consequential decisions emerged or were proclaimed and in which traditions were enacted and passed on. In such conversations, memorable interchanges took place spontaneously, without generally being planned or even in contrast to intentions brought to the table. How did the physical and social setting of the occasion open up a space across the tabletop in which discourses could form of their own volition? How did things come to word, which none of the participants had in mind beforehand or would have come up with on their own?

Several years ago in the introduction to an issue of this journal (Stahl 2007), we imagined a group of people meeting around a primordial tribal fire (see Fig. 1). They discussed the dialectical relationship between the fire as a spiritual phenomenon and the individual $\operatorname{logs}$ that contributed to its continuing existence. Participants in the discussion exchanged questions and perspectives, building a multi-vocal network of utterances that reflected the complexity of the relationship.

The discussion of the emergence of the tribal fire from the burning logs served as a metaphor for the problem of the relationship of group cognition to individual thinking. The questions and reflections of the tribal members mirrored theories influential in CSCL research, from mental models to distributed cognition, activity theory, and actor-network theory. The articles in that issue of the journal exemplified various positions within CSCL analogous to those in the mythical discourse of the tribe.

Conversation around the fire was paradigmatic of oral society. The answering of questions and the relating of narratives were knowledge-disseminating and knowledgebuilding mechanisms for unmediated verbal interaction. In literate society, texts like professional journals can open analogous "spaces" in which knowledge can be built and archived for communities such as research fields. In the digital age, technologies like wikis can play a similar role, at least in theory.

Imagine a mature Wikipedia page that presents a fairly coherent view, with a subtle intertwining of numerous thematic threads. Assume that the page has grown through a

G. Stahl $(\bowtie)$

Drexel University, Philadelphia, USA

e-mail: Gerry@ijCSCL.org 

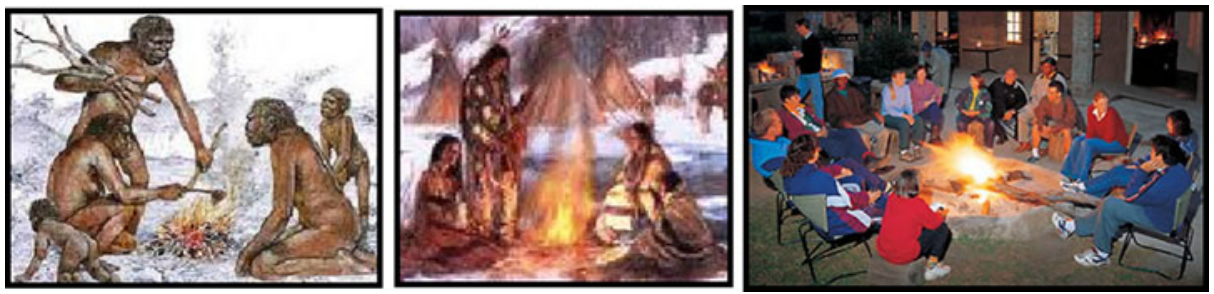

Fig. 1 Tribal fires throughout human history

patchwork of edits by many, essentially anonymous contributions. When you read the page, you get a comprehensible impression of a complex idea that far exceeds what any of the individual contributors had in mind when they edited the page. Probably, most of the contributors tweaked details of specific sections that were in the wiki page at the time, and few if any contributors worried about the overall shape and impact of the page as a whole. The page emerged from this complex, unplanned, willy-nilly process of collaborative meaning making. The meaning of the final wiki page is a function of the interplay of the many words and sentences in the current version. While this web of meaning is the result of individual actions, it does not correspond to the thought of any one individual, nor is it a simple combination of such thoughts. It is the residue from a sequential interaction that was not planned but just happened. The wiki page - as a persistent, observable artifact - makes visible the nature of the groupgenerated meaning as a semantic web resulting from the unplanned, intentional, sequential interactions of individuals, which is not attributable to the agency of any individual contributor.

Recently, the availability of "tabletop" educational environments has raised the possibility of a digital technology that can serve as the center for small groups of learners to engage in a hybrid of oral, literate, and digital interaction: a multimedia tribal fire for the classroom, workplace, or social gathering. It seems that the tabletop can integrate physical and digital artifacts, spoken and written texts, and human and computational support to form a focal point, subject matter, and dynamic resource for collaborative knowledge building. However, if the field of CSCL has learned anything in the past two decades, it is that apparent technological possibilities require considerable interface design, user evaluation, pedagogical structuring, and collaborative culture in order to achieve desirable educational results.

This leads to the need for a flash theme of tabletop computing. A "flash theme" is a topic that has flared up in the current CSCL research community as an issue of timely importance and as a matter of viral concern. In previous years, we have featured articles on the flash themes of community-based learning, scripting in CSCL, argumentation in CSCL, and methods for evaluating CSCL. In this issue, we begin a flash theme on tabletop interfaces for CSCL. Like most of the earlier flash themes, this one flamed up at a workshop of CSCL researchers. The participants of the workshop and others have prepared scholarly presentations about the relevance of tabletop interfaces to the support of collaborative learning. We begin with several introductory papers in this issue. We anticipate more papers next year, and welcome additional submissions on this flash theme.

\section{In this issue}

We begin with an overview of the flash theme, interactive tabletops in education, by Pierre Dillenbourg and Michael Evans. This paper addresses in some detail the temptation to over-generalize the potential of the technology itself to produce educational gains and the 
tendency for inflated expectations based on the nature of the tabletop medium. The paper first provides a description of tabletop interfaces and their most common components, illustrating with examples of diverse research prototypes. It then discusses the primary characteristics of tabletops that lend them a socio-cultural flavor, at least potentially: they provide a hands-on multi-modal medium for co-located interaction within small groups. So tabletops tend to be small-group environments, in contrast both to the personal style of desktops, laptops, or mobile devices and to the public or classroom style of whiteboards. The role of small-group tool, mediating between individual cognition and classroom practices raises a number of issues for CSCL research on tabletop computing. The paper enumerates 33 such issues, clustered into the circles of: interaction with the individual student, support for small-group interaction, classroom orchestration, and institutional context. These dimensions highlight the complexity of tabletop interaction and militate against the tendency to assume that it is simply more "natural" than interaction with laptops because of its support for direct physical gestures. The paper provides a tentative catalog of central topics for analyzing tabletops in future articles of the flash theme.

The overview of research in tabletop environments for CSCL continues with a synthetic review of the literature by Steven E. Higgins, Emma Mercier, Liz Burd, and Andrew Hatch. They build on the preceding introduction and further develop the typology of issues for research, organizing over a hundred studies into categories related to the nature of the multi-touch interactive tabletop's surface, how it can be touched by its users, how it is networked with other devices, or how the tabletop is used to support collaborative learning. This review of the early literature on tabletops not only summarizes initial findings, but more importantly sketches the territory to be covered by needed future research. A central concern is how the tabletop design affords particular interaction patterns among the students using it together.

The next paper, by Sara Price and Taciana Pontual Falcão, takes up the task of analyzing interaction patterns that emerge during use of a tabletop interface in England. It focuses on the productive role of interference-for example when two users simultaneously move tabletop objects in a way that interferes with each other. This seems related to recent ijCSCL discussions of "productive failure" (Kapur and Kinzer 2009; Pathak et al. 2011; Wang et al. 2011) - in which engagement with what Piaget might consider cognitive conflict leads to collaborative learning. Building on ideas developed in earlier flash-theme discussions of argumentation and scripting, this paper analyzes instances of interference in usage of tabletops for collaborative learning. The tabletop environment provides a rich space for several students to explore phenomena of physics collaboratively. The open, embodied, multi-touch micro-world allows for many forms of student-centered discovery and small-group interaction, as well as multiple opportunities for interference of both actions and conceptualizations. The paper systematically looks into the variety and consequences of such interference. While the tabletop interface affords multiple forms of interference, it is the nature of the small-group collaboration processes that primarily influences the learning that results. Productive argumentation involves the students in seriously considering each other's perspectives and resolving conflicts to allow a group solution. Determining how this takes place involves rather detailed analysis of the physical and verbal interactions around the tabletop.

Tabletop equipment is prohibitively expensive today for deployment beyond research settings. A group in Chile, including Eyal Szewkis, Miguel Nussbaum, Tal Rosen, Jose Abalos, Fernanda Denardin, Daniela Caballero, Arturo Tagle and Christian Alcohoiado, has been exploring a relatively inexpensive alternative: allowing a large group - a whole classroom - of students to share a projected computer display in common and each have 
access to interacting with it using multiple mice. A number of classroom practices are instituted, involving mouse-based interaction patterns; they implement what the authors call "silent collaboration" exchanges in which students work together on the assigned task without talking. The exchanges of computer icons by the student dyads using their mice are displayed for the dyads and for the whole class on a projection screen. In this way, all the students can be simultaneously actively involved in collaborative-learning activities within a large group, gathered around a shared display. The experimental results indicate significant collaborative-learning gains with this approach.

Telling stories is a fundamental form of interaction in oral societies; we learn how to create and narrate stories as young children (Bruner 1990; Ong 1998). In an experiment involving scripted collaboration and computer support, Giulia Geimini-Hornsby, Shaaron Ainsworth, and Claire O'Malley investigate how asking questions can help to drive the development of storytelling skills. They find the "guided-reciprocal-questioning script" to be effective in a number of ways. Interestingly, this is a very flexible kind of script, in contrast to many scripts investigated in CSCL research, allowing students to select what question to pose and even motivating them to formulate their own questions. Also, as a form of scaffolding, the use of this script seems to continue to exert a positive influence after the use of the script is withdrawn.

The next paper also explores the effect of scripted questioning. In a controlled experiment by Inge Molenaar, Ming Ming Chiu, Peter Sleegers, and Carla van Boxtel, triads of students engaged in collaborative learning in the Netherlands are periodically interrupted by questions on their computer. In the three conditions, they are shown either just a cartoon drawing of a teenage boy (the avatar), the drawing along with instructions on some action to take and suggestions on how to take it (the "suggestive" scaffold), or the drawing with a question about how to take some action and a text box for typing in a response (the "problematizing" scaffold). Analysis of the results indicates that the students shown the problematizing scaffolds learned the most domain knowledge. The questions were timed to correspond to points in the collaborative work when students would be thinking about their collaborative-learning process (engaging in metacognition). By prompting their metacognition in a timely manner, the scaffolds apparently aided the students in their reflections and interactions.

We close this issue and the volume with an exploration of community building by Donatella Cesareni, Francesca Martini, and Ilaria Mancini. They report on activities that took place in Italy during the second year of a European CSCL project-I happened to be involved in the system design phase in the first year in Germany (Stahl 2006, chapters 7 \& 8). In contrast to the carefully structured classrooms of young students in the preceding papers, here we have a reflection on a relatively free-flowing community of teachers, researchers, and university students: interacting online and face-to-face, synchronously and asynchronously, in text and through speech. For most participants, this was an initial involvement in what must be considered the early days of CSCL, given the state of computer support and experience in that setting. This helped to make visible the dialectic between expert and novice as the community matured.

\section{Six years of ijCSCL}

This issue completes six incredibly successful years of publication of the journal. The journal has attracted many important submissions and has served the CSCL research community by publishing a broad range of papers covering new ideas, rigorous studies, 
strong theoretical reflections, methodological innovations, and insightful reports. It not only serves as an archive for significant findings, but also as a venue for reflection upon the theories, methodologies, and agendas of the global CSCL research field, providing insights into the nature of collaborative learning as well as practical suggestions for implementing computer support. The quality of the published articles is due to the Board of Editors and other reviewers, who have not only selected the papers to be printed, but also provided key suggestions to the authors, which have resulted in substantial improvements to the final versions; they have kept the flames of this tribal fire burning brightly.

For next year, there will be some changes to the Board of Editors due to a substantial increase in the number of submissions to the journal. All Board members were asked if they wanted to renew their Board membership for another 4 years. Of 72 Board members, $82 \%$ committed to serving further and 13 decided to rotate off the Board to allow new members to join. In addition, the Associate Editor positions have rotated; the 6 new Associates will be taking on the supervision of reviews and the drafting of the meta-reviews, along with the Executive Editors. Nancy Law joins as an additional Executive Editor. Carol Chan, Ulrike Cress, Manu Kapur, Sten Ludvigsen, Carolyn Rosé, and Daniel Suthers take on the expanded Associate Editor roles.

At this time, the journal Executive Editors would like to express our sincere thanks to the people stepping off the Board, for their 6 years of support, helping to get the journal off the ground. We are also grateful to the former Associate Editors-who will all be continuing on the Board-for their leadership during this critical period. As always, we recognize the people who have contributed the decisive reviews, including the following:

Shaaron Ainsworth, Rick Alterman, Jerry Andriessen, Nancy Ares, Baharuddin Aris, Hans Christian Arnseth, Maarit Arvaja, Christa Asterhan, Maria Avgerinou, Gerardo Ayala, Michael Baker, Maria Bannert, Liam Bannon, Ulrika Bennerstedt, Johanna Bluemink, Daniel Bodemer, Jacqueline Bourdeau, Paul Brna, Bertram Bruce, Amy Bruckman, Jurgen Buder, Murat Cakir, Angela Carell, John Carroll, Carol Chan, Rosanna Chan, Tak-Wai Chan, Elizabeth Charles, Clement Chau, Fei-Ching Chen, Britte Cheng, Cesar Collazos, Ulrike Cress, Charles Crook, Lucilla Crosta, Ton de Jong, Anne Meier Deiglmeyer, Muhammet Demirbilek, Sharon Derry, Bram DeWever, Pierre Dillenbourg, Angelique Dimitrakopoulou, Lone Dirckinck-Holmfeld, Nina Dohn, Gilles Doiron, Paul Dourish, Nathan Dwyer, Anna Engel, Noel Enyedy, Gijsbert Erkens, Michael A Evans, Deller Ferreira, Frank Fischer, Brian Foley, Andrea Forte, Hugo Fuks, Andreas Gegenfurtner, Anne Gerdes, Sean Goggins, Ricki Goldman, Luisa Aleyda Gonzalez, Begoata Gros, Jonathan Grudin, Frode Guribye, Joerg Haake, Paivi Hakkinnen, Kai Hakkarainen, Raija Hamaalainen, Andreas Harrer, Wu He, Libby Hemphill, Thomas Herrmann, Friedrich Hesse, Steven Higgins, Cindy Hmelo-Silver, Christopher Hoadley, Ulrich Hoppe, Christine Howe, Tien-Chu Huang, James Hudson, Diane Hui, Chris Hundhausen, Liisa Ilomaki, Shahrinaz Ismail, Isa Jahnke, Sanna Jarvela, Patrick Jermann, Richard Joiner, Christopher Jones, Robert Jorczak, Regina Jucks, Yael Kali, Victor Kaptelinin, Manu Kapur, Anastasios Karakostas, Fengfeng Ke, Diane Jass Ketelhut, Andrea Kienle, Joachim Kimmerle, Paul Kirschner, Lars Kobbe, Matthew Koehler, Timothy Koschmann, Ingeborg Krange, Eleni Kyza, Therese Laferriere, Minna Lakkala, Victor Lally, Niki Lambropoulos, Mary Lamon, Yu-Ju Lan, Johann Larusson, Nancy Law, Mark Lee, Erno Lehtinen, Maria Ligorio, Kenneth Lim, Robb Lindgren, Oskar Lindwall, Lasse Lipponen, Geoffrey Liu, Jia-Jiunn Lo, Jacques Lonchamp, Chee-Kit Looi, Jingyan Lu, Rose Luckin, Sten R. Ludvigsen, Andreas Lund, Kristine Lund, Johan Lundin, Kim MacKinnon, Alejandra Martinez, Richard Medina, Monika Mital, Naomi Miyake, Anders Morch, Johannes Moskaliuk, Daisy Mwanza-Simwami, Bonnie Nardi, Brian Nelson, Bernhard Nett, Matthias Nackles, 
E. Michael Nussbaum, Angela O'Donnell, John O'Donoghue, Claire O'Malley, Hiroaki Ogata, Javier Onrubia, Jun Oshima, Khaziyati Osman, Roy Pea, Ruediger Pfister, Manoli Pifarre, Sara Price, Mingzhu Qiu, Subba Rao, Ingvil Rasmussen, Janet Read, Peter Reimann, Ann Renninger, Jochen Rick, Alan Roberts, Tim Roberts, Jennifer Rode, Markus Rohde, Jeremy Roschelle, Carolyn Rose, Liam Rourke, Nikol Rummel, Nadira Saab, Roger Saljo, Johann Sarmiento-Klapper, Claudia Sassenrath, Tammy Schellens, Oliver Scheuer, Gregg Schraw, Baruch Schwarz, Anna Sfard, David Shaffer, Wesley Shumar, Amy Soller, Nancy Songer, Hans Spada, Marc Stadtler, Gerry Stahl, Karsten Stegmann, Constance Steinkuehler, Alan Stevenson, Jan-Willem Strijbos, Masanori Sugimoto, Daniel Suthers, Berthel Sutter, Seng-Chee Tan, Steven Tanimoto, Gustav Taxen, Pierre Tchounikine, Meng Yew Tee, Chris Teplovs, Ramon Prudencio Toledo, Stefan Trausan-Matu, Jan van Aalst, Ravi Vatrapu, Marjaana Veermans, Sarah Walter, Jim Waters, Christof Wecker, Rupert Wegerif, Armin Weinberger, Gordon Wells, Martin Wessner, Tobin White, Donghee Wohn, Volker Wulf, Fatos Xhafa, Ling Ling Yen, Jennifer Yeo, Fu-Yun Yu, Nicola Yuill, Joyce Yukawa, Coco Zhao, Nan Zhou.

\section{References}

Bruner, J. (1990). Entry into meaning. In Acts of meaning. (pp. 67-97): Harvard U Press.

Kapur, M., \& Kinzer, C. K. (2009). Productive failure in CSCL groups. International Journal of ComputerSupported Collaborative Learning, 4(1), 21-46.

Ong, W. (1998). Orality and literacy: The technologizing of the world. New York: Routledge.

Pathak, S. A., Kim, B., Jacobson, M. J., \& Zhang, B. H. (2011). Learning the physics of electricity: A qualitative analysis of collaborative processes involved in productive failure. International Journal of Computer-Supported Collaborative Learning., 6(1), 57-73.

Stahl, G. (2006). Group cognition: Computer support for building collaborative knowledge. Cambridge, MA: MIT Press. $510+$ viii pages.

Stahl, G. (2007). CSCL and its flash themes. International Journal of Computer-Supported Collaborative Learning., 2(4), 359-362.

Wang, H.-C., Rosé, C. P., \& Chang, C.-Y. (2011). Agent-based dynamic support for learning from collaborative brainstorming in scientific inquiry. International Journal of Computer-Supported Collaborative Learning., 6(3), 371-395. 